\title{
Deep inelastic scattering on a nucleus using holography
}

\author{
Kiminad A. Mamo* and Ismail Zahed ${ }^{\dagger}$ \\ Department of Physics and Astronomy, Stony Brook University, Stony Brook, New York 11794-3800, USA
}

(Received 4 June 2019; published 22 August 2019)

\begin{abstract}
We consider deep inelastic scattering (DIS) on a nucleus described using a density expansion. In leading order, the scattering is dominated by the incoherent scattering on individual nucleons distributed using the Thomas-Fermi approximation. We use the holographic structure functions for DIS scattering on single nucleons to make a nonperturbative estimate of the nuclear structure function in leading order in the density. Our results are compared to the fits from data in the large-x regime.
\end{abstract}

DOI: 10.1103/PhysRevD.100.046015

\section{INTRODUCTION}

Many years ago, the EMC Collaboration observed that the structure function of iron differs substantially from that of the deuteron. This observation was later supported by dedicated unpolarized deep inelastic scattering (DIS) experiments from several collaborations at CERN, SLAC and FNAL [1-4]. The EMC observation was of course surprising. Why would scattering at high energy and momentum transfer be affected by intranuclear effects that are much lower in energy?

The large body of empirical DIS scattering on nuclei points at the enhancement of incoherent scattering in DIS, whereby two or more nucleons act coherently to produce sizable deviations from incoherent scattering as the sum of DIS scattering over the individual nuclear structure functions. This is best seen in the low-x region with the depletion of the structure functions also referred to as shadowing [1,2]. In the large-x region, nuclear effects such as binding and Fermi motion are more pronounced [3].

The purpose of this paper is to examine the role of strong coupling when the nuclear many-body system is probed electromagnetically in the DIS limit. Since QCD is approximately conformal both at strong and weak coupling, satisfying various scaling laws, it is important that the issues of kinematics (conformal symmetry) are separated from issues of dynamics (asymptotic freedom and confinement). For that, we organize the DIS scattering amplitude on a nucleus in terms of DIS scattering amplitudes on one, two, ... nucleons in a nuclear medium where the individual nucleons are distributed using the Thomas-Fermi

\footnotetext{
*kiminad.mamo@stonybrook.edu

ismail.zahed@stonybrook.edu
}

Published by the American Physical Society under the terms of the Creative Commons Attribution 4.0 International license. Further distribution of this work must maintain attribution to the author(s) and the published article's title, journal citation, and DOI. Funded by SCOAP ${ }^{3}$. approximation. For dilute nuclei with small atomic number $A$, the leading contribution is on one-nucleon state smeared by Fermi motion which should be justified in the large-x region. Each of the DIS scattering on the few-nucleon amplitudes is then estimated using holography.

In the holographic limit, DIS scattering on a spin- $\frac{1}{2}$ state reveals that the scattering is hard and nucleonic instead of partonic [5]. In the double limit of a large number of colors and strong gauge coupling, the short distance correlations of the electromagnetic current are dominated by doubletrace operators which are hadronic. The partonic operators develop large anomalous dimensions as they carry color and radiate strongly. Their energy is quickly depleted before they are struck, leaving only the colorless hadronic structures to scatter off, i.e., the nucleon and its pion cloud. This description of DIS scattering fits well with the nuclear description of a nucleus as an assembly of individual nucleons dressed with pion clouds and bound mostly by two-body forces.

The organization of the paper is as follows: In Sec. II we briefly introduce the key elements in DIS scattering on a nucleus. We make use of a density expansion and the Thomas-Fermi approximation to describe the leading contributions. In Sec. III the nucleus structure function in leading order in the density expansion is derived, making explicit the role of binding and Fermi motion. We use the holographic results for DIS scattering on a single nucleon to evaluate the pertinent R-ratio in leading order of the density. The results are compared to the empirical R-ratio for light nuclei. Our conclusions are in Sec. IV.

\section{FINITE NUCLEUS}

In a DIS process on a nucleus, a virtual photon of 4-momentum $q$ scatters off a nucleus of 4-momentum $P_{A}$ producing a complex set of hadronic final states. The inclusive cross section sums over all these final states. Comprehensive descriptions of this process can be found in [6], to which we refer for more details. For unpolarized 
scattering on a nucleus, the DIS tensor is given by the response function

$$
\mathcal{G}_{A}^{\mu \nu}=i \int d^{4} y e^{i q \cdot y}\left\langle P_{A}\left|\left[J^{\mu}(y), J^{\nu}(0)\right]\right| P_{A}\right\rangle,
$$

where $J$ is the electric current. $\mathcal{G}$ follows the general tensor decomposition (mostly positive metric)

$$
\begin{aligned}
\mathcal{G}_{A}^{\mu \nu}= & F_{1}^{A}\left(x_{A}, q^{2}\right)\left(\eta^{\mu \nu}-\hat{q}^{\mu} \hat{q}^{\nu}\right) \\
& +\frac{2 x_{A}}{q^{2}} F_{2}^{A}\left(x_{A}, q^{2}\right)\left(P_{A}^{\mu}+\frac{1}{2 x_{A}} q^{\mu}\right)\left(P_{A}^{\nu}+\frac{1}{2 x} q^{\nu}\right)
\end{aligned}
$$

with manifest current conservation. $F_{1,2}^{A}$ are the nucleus structure functions expressed in terms of Bjorken $x_{A}=$ $-q^{2} / 2 q \cdot P_{A}$ with a virtual photon momentum $q=(\omega, 0,0, q)$. In the DIS kinematic we take $\omega \approx q$ with large $q^{2} \rightarrow \infty$ but fixed $x$. In the nucleus rest frame $P_{A}=$ $\left(A\left(m_{N}-B\right), 0,0,0\right)$ where $B=8.5 \mathrm{MeV}$ is the binding energy per nucleon, so that

$$
\frac{x}{x_{A}}=A\left(1-\frac{B}{m_{N}}\right)
$$

[(2.1) and (2.2)] is related to the forward part of the virtual Compton scattering amplitude by the optical theorem. More specifically, the forward Compton scattering amplitude is

$$
\mathcal{T}_{A}^{\mu \nu}=i \int d^{4} y e^{i q \cdot y}\left\langle P_{A}\left|T^{*} J^{\mu}(y) J^{\nu}(0)\right| P_{A}\right\rangle
$$

with a similar tensor decomposition

$$
\begin{aligned}
\mathcal{T}_{A}^{\mu \nu}= & \tilde{F}_{1}^{A}\left(x_{A}, q^{2}\right)\left(\eta^{\mu \nu}-\hat{q}^{\mu} \hat{q}^{\nu}\right) \\
& +\frac{2 x_{A}}{q^{2}} \tilde{F}_{2}^{A}\left(x_{A}, q^{2}\right)\left(P_{A}^{\mu}+\frac{1}{2 x_{A}} q^{\mu}\right)\left(P_{A}^{\nu}+\frac{1}{2 x_{A}} q^{\nu}\right) .
\end{aligned}
$$

The structure functions satisfy $F_{1,2}^{A}=2 \pi \operatorname{Im} \tilde{F}_{1,2}^{A}$.

\section{A. Density expansion}

Ignoring Pauli blocking, we can assess Eq. (2.1) using a density expansion in terms of stable nucleon states by averaging the forward Compton amplitude over a complete set of stable one-nucleon, two-nucleon, ... states distributed in a finite nucleus. If we denote by

$$
\left\langle P_{A} \mid P_{A}\right\rangle=(2 \pi)^{3} 2 E_{A} \delta\left(\overrightarrow{0}_{A}\right) \equiv 2 E_{A} V_{3}
$$

the scattering normalization of the (finite) nucleus, then we may expand Eq. (2.1) in powers of the density
$\frac{\mathcal{G}_{A}^{\mu \nu}}{\left\langle P_{A} \mid P_{A}\right\rangle}=\int d N \mathcal{G}_{N}^{\mu \nu}+\frac{1}{2 !} \int d N_{1} d N_{2} \mathcal{G}_{2 N}^{\mu \nu}+\cdots$

The connected DIS amplitudes are

$$
\begin{aligned}
\mathcal{G}_{n N}^{\mu \nu}= & i \int d^{4} z e^{i q \cdot z} \\
& \times\left\langle N\left(p_{1}\right) \ldots N\left(p_{n}\right)\left|\left[J^{\mu}(z), J^{\nu}(0)\right]\right| N\left(p_{1}\right) \ldots N\left(p_{n}\right)\right\rangle_{c}
\end{aligned}
$$

with the nucleon phase-space occupation factors

$$
d N_{i}=4 \frac{d^{3} r_{i}}{V_{3}} \frac{d^{3} p_{i}}{(2 \pi)^{3}} \frac{1}{2 E_{p_{i}}} \mathbf{n}\left(r_{i}, p_{i}\right)
$$

for unpolarized neutrons and protons. Each of the nucleon in Eq. (2.8) is on mass-shell modulo binding (see below) with a 4-momentum $p_{i}=\left(E_{p_{i}}, \vec{p}_{i}\right)$. A similar expansion at finite temperature using pions was successfully used for electromagnetic emissivities from heavy ion collisions at collider energies [7].

The leading contribution in Eq. (2.7) involves the forward Compton amplitude on a single nucleon averaged over the nucleus, and amounts to the totally incoherent contribution to the structure functions. The next-to-leading order contribution corresponds to forward Compton scattering on a pair of nucleons which is the first coherent correction to the leading contribution. As most nuclei are well described by trapped nucleons in a mean-field potential with mostly two-body interactions, the dominant contributions in the expansion (2.7) are the leading and next-to-leading order.

\section{B. Thomas-Fermi approximation}

The distribution of nucleons in a nucleus is uniform over a range $r<R_{A}$ up to a surface thickness $\epsilon_{A}=\delta / R_{A} \ll 1$ for large nuclei, so that the nucleon density distribution can be approximated by

$$
\begin{aligned}
\rho_{A}(r)= & \rho_{0} \theta\left(R_{A}-r\right)+\rho_{0}\left(1-\frac{\left(r-R_{A}\right)}{\delta}\right) \\
& \times \theta\left(r-R_{A}\right) \theta\left(R_{A}+\delta-r\right) .
\end{aligned}
$$

For infinitely large nuclei or nuclear matter $\rho_{0}=$ $0.17 \mathrm{fm}^{-3} \cdot R_{A}$ is fixed by the normalization of the density (2.10) to $A$. Typically, for nuclei with $A \geq 12, R_{A}=R_{0} A^{\frac{1}{3}}$, $R_{0}=1.12 \mathrm{fm}$ and the surface thickness $\delta=2.4 \mathrm{fm}$. We now assume the nucleus to be a degenerate Fermi gas of nucleons trapped in a finite well of depth $V_{0}<0$, with a Fermi momentum $p_{F}(r)$ fixed by the density $\rho_{A}(r)$ using the Thomas-Fermi approximation for symmetric nuclei,

$$
\rho_{A}(r)=\frac{4}{(2 \pi)^{3}} \frac{4 \pi}{3} p_{F}^{3}(r) .
$$


For uniform (nuclear) matter with $\rho_{0}=0.17 \mathrm{fm}^{-3}$, the Fermi momentum is $p_{F}=268 \mathrm{MeV}$, and the typical kinetic energy per nucleon is $K=23 \mathrm{MeV}$ so that the well depth is $V_{0}=-K-2 B=-40 \mathrm{MeV}$ ( $2 B$ is the binding energy ignoring surface and symmetry contributions). The occupation number in Eq. (2.9) is then $\mathbf{n}(r, p)=\theta\left(p_{F}(r)-|\vec{p}|\right)$.

\section{NUCLEUS STRUCTURE FUNCTIONS}

High energy photon-nucleus scattering shows that the photonuclear cross sections scale as $\sigma_{\gamma A} \approx A^{0.92} \sigma_{\gamma N}$ for $\omega>$ $3 \mathrm{GeV}$ [8]. In this regime the scattering is off the nuclear volume that scales like $A$ and should describe well the large-x region. For small $\mathrm{x}$, the virtual photon acts as a colorless dipole. High energy dipole-nucleus scattering is equivalent to hadron-nucleus scattering with cross sections that scale like $\sigma_{N A} \approx A^{0.8} \sigma_{N N}$ for $\sqrt{s} \approx(10-25) \mathrm{GeV}$ [9], which is mostly off the nuclear edge as it scales like $A^{\frac{2}{3}}$. Both volume and surface effects are included in our expansion using the Thomas-Fermi approximation.

\section{A. Leading density contribution}

Since in leading order the coherent scattering off two nucleons or more is absent, we expect this contribution to describe well the large-x region. With this in mind, the leading density contribution in Eq. (2.7) is readily reduced using Eqs. (2.10) and (2.11):

$$
\begin{aligned}
\frac{\mathcal{G}_{A}^{\mu \nu}}{\left\langle P_{A} \mid P_{A}\right\rangle} \approx & \rho_{0} \frac{4 \pi}{3} R_{A}^{3} \int \frac{d^{3} p}{2 V_{3} E_{p}} \frac{\theta\left(p_{F}-|\vec{p}|\right)}{\frac{4}{3} \pi p_{F}^{3}} \mathcal{G}_{p}^{\mu \nu} \\
& +16 \pi \int_{R_{A}}^{R_{A}+\Delta} r^{2} d r \int \frac{d^{3} p}{(2 \pi)^{3}} \frac{1}{2 V_{3} E_{p}} \\
& \times \theta\left(p_{F}(r)-|\vec{p}|\right) \mathcal{G}_{p}^{\mu \nu} .
\end{aligned}
$$

The DIS scattering on a single nucleon $\mathcal{G}_{p}^{\mu \nu}$ in Eq. (3.1) can be decomposed similarly to Eq. (2.5):

$$
\begin{aligned}
\mathcal{G}_{p}^{\mu \nu}= & F_{1}^{p}\left(x_{p}, q^{2}\right)\left(\eta^{\mu \nu}-\hat{q}^{\mu} \hat{q}^{\nu}\right) \\
& +\frac{2 x_{p}}{q^{2}} F_{2}^{p}\left(x_{p}, q^{2}\right)\left(p^{\mu}+\frac{1}{2 x_{p}} q^{\mu}\right)\left(p^{\nu}+\frac{1}{2 x_{p}} q^{\nu}\right) .
\end{aligned}
$$

The nucleon 3-momentum is fixed by Fermi motion with $x_{p}=-q^{2} / 2 q \cdot p$ and tied to $x$ by

$$
\frac{x}{x_{p}}=\frac{E_{p}}{m_{N}}-\frac{|\vec{p}|}{m_{N}} \cos \theta_{p}
$$

Here $x=-q^{2} / 2 \omega m_{N}$ is Bjorken-x for a free nucleon at rest. The first contribution in Eq. (3.1) is due to the uniform density of the nucleus in bulk and is of order $A$, while the second contribution arises from the surface of the nucleus and is of order $A^{\frac{2}{3}}$, with the estimate

$$
\frac{3 \kappa_{A}}{2} \rho_{0} \frac{4 \pi}{3} R_{A}^{3} \int \frac{d^{3} p}{2 V_{3} E_{p}} \frac{\theta\left(p_{S}-|\vec{p}|\right)}{\frac{4}{3} \pi p_{S}^{3}} \mathcal{G}_{p}^{\mu \nu} .
$$

The mean surface Fermi momentum $p_{S}$ is fixed by Eq. (2.11) with a mean surface density approximated by $\frac{1}{2} \rho_{0}$, Here $\kappa_{A}=\kappa \epsilon_{A}$ with $\kappa$ adjusting for this approximation. The dominant correction to Eq. (3.1) stems from the nucleon pair or two-body correlations in Eq. (2.7), as three- and higher-body correlations are known to be small in a nucleus.

Inserting Eqs. (3.1) and (3.2) into Eq. (2.7) leads to the nucleus structure functions in leading order in the density

$$
\begin{aligned}
F_{2}^{A}\left(x_{A}, q^{2}\right) \approx & \rho_{0} \frac{4 \pi}{3} R_{A}^{3}\left[\left(\int d^{3} p \frac{E_{A}}{E_{p}} \frac{\theta\left(p_{F}-|\vec{p}|\right)}{\frac{4}{3} \pi p_{F}^{3}}+\frac{3 \kappa_{A}}{2} \int d^{3} p \frac{E_{A}}{E_{p}} \frac{\theta\left(p_{S}-|\vec{p}|\right)}{\frac{4}{3} \pi p_{S}^{3}}\right)\right. \\
& \left.\times\left(\frac{\left(p+\frac{q}{2 x_{p}}\right)^{2}-3\left(P_{A} \cdot p-\frac{q^{2}}{4 x_{A} x_{p}}\right)^{2}\left(P_{A}^{2}-\frac{q^{2}}{4 x_{A}^{2}}\right)^{-1}}{\left(P_{A}+\frac{q}{2 x_{A}}\right)^{2}-3\left(P_{A}^{2}-\frac{q^{2}}{4 x_{A}^{2}}\right)}\right) \frac{x_{p}}{x_{A}} F_{2}^{p}\left(x_{p}, q^{2}\right)\right],
\end{aligned}
$$

and similarly for $F_{1}^{A}$. Given the nucleon structure function $F_{2}^{p}$, Eq. (3.5) is the leading order estimate for the nucleus structure function $F_{2}^{A}$. We now choose to analyze Eq. (3.5) using the holographic results for the nucleon structure function.

\section{B. Holographic nucleon structure function}

DIS scattering at strong coupling $\lambda=g^{2} N_{c}$ on a nucleon using the holographic construction was carried initially by Polchinski and Strassler [5] and others [10]. In brief, the metric in a slab of five-dimensional anti-de Sitter space $\left(\mathrm{AdS}_{5}\right)$ is given by

$$
d s^{2}=\frac{R^{2}}{z^{2}}\left(\eta_{\mu \nu} d y^{\mu} d y^{\nu}+d z^{2}\right)
$$

with a fixed wall at $z_{H}$. The bulk $\operatorname{AdS}_{5}$ radius $R$ and the string length $l_{s}$ are related to the boundary gauge coupling $\lambda=R^{4} / l_{s}^{4} \gg 1$. In holography, Compton scattering on a 
nucleon at the boundary maps onto the scattering in bulk of the R-current onto a dilatino with spin- $-\frac{1}{2}$ at large $\mathrm{x}$, while at small $x$ the same scattering is dominated by the t-exchange of a closed string, with the interpolating result [5]

$F_{2}^{p}\left(x, q^{2}\right)=\tilde{\mathbb{C}}\left(\frac{m_{N}^{2}}{-q^{2}}\right)^{\tau-1}\left(x^{\tau+1}(1-x)^{\tau-2}+\mathbb{C}\left(\frac{m_{N}^{2}}{-q^{2}}\right)^{\frac{1}{2}} \frac{1}{x^{\Delta_{\mathbb{P}}}}\right)$.

Here $\tau=\Delta-\frac{1}{2}$ refers to the twist, and $\Delta_{\mathbb{P}}=2\left|1-\Delta^{2}\right| /$ $\sqrt{\lambda} \ll 1$. Also, $\Delta=m R+2$ is the conformal dimension of the spin $-\frac{1}{2}$ field, and $\tilde{\mathbb{C}}, \mathbb{C}$ are two independent constants. We have expressed $z_{H}$ in units of $m_{N}$. The holographic nucleon structure function for the soft wall model reproduces Eq. (3.7) at large $q^{2}$ [11].

For $m R=\frac{3}{2}$ or $\tau=3$, the structure function (3.7) obeys conformal scaling, i.e., $\left(1 / q^{2}\right)^{2}$. We recall that at strong coupling, conformal scaling is at the origin of the hard scaling law [12] for the nucleon form factor. In contrast and at weak coupling, the structure function obeys Bjorken scaling (independent of $q^{2}$ ) with $F_{2}^{p}\left(x, q^{2}\right) \approx \sqrt{x}(1-x)^{3}$ at the nucleon mass scale. The $\sqrt{x}$ behavior for small $\mathrm{x}$ is conform with the Kuti-Weisskopf rule for nonsinglet structure functions [13].

In Fig. 1 we show the x-dependent part of the nucleon structure function at weak coupling (dashed curve) and strong coupling (solid curve) normalized to 1 . The former is peaked towards low $\mathrm{x}$, and even further after the expected Dokshitzer-Gribov-Lipatov-Altarelli-Parisi evolution. The latter is skewed towards $x=1$, which reflects the fact that in the double limit of large $N_{c}$ and strong coupling $\lambda$, DIS scattering is off the nucleon as a whole. The leading twists are nonperturbative and of order $\lambda^{0}$. They arise from doubletrace operators which are hadronic and not partonic [5].

\section{R-ratio}

It is customary to analyze DIS scattering on a nucleus through the R-ratio defined as

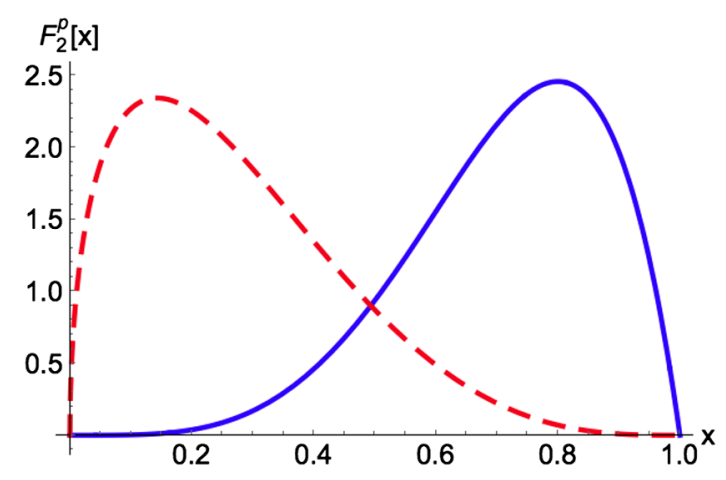

FIG. 1. Large-x dependence of the nucleon structure function $F_{2}^{p}[x]$ for weak coupling (dashed curve) and strong coupling (solid curve) normalized to 1 .

$$
R\left[x, q^{2}\right]=\frac{\frac{1}{A} F_{2}^{A}\left(x, q^{2}\right)}{F_{2}^{p}\left(x, q^{2}\right)}
$$

and expressed in terms of Bjorken-x for large but fixed $q^{2}$. Note that $x_{p, A}$ translate to $x$ through Eqs. (2.3) and (3.3). With this in mind, the explicit expression for Eq. (3.8) is

$$
\begin{aligned}
R\left[x, q^{2}\right] \approx & \int \frac{d^{3} p}{1+3 \epsilon_{A}}\left[\left(\frac{\theta\left(p_{F}-|\vec{p}|\right)}{\frac{4}{3} \pi p_{F}^{3}}+\frac{3 \kappa_{A}}{2} \frac{\theta\left(p_{S}-|\vec{p}|\right)}{\frac{4}{3} \pi p_{S}^{3}}\right)\right. \\
& \times \frac{3 x_{p} E_{A}}{2 x_{A} E_{p}}\left(\left(\frac{E_{A} E_{p}+\frac{-q^{2}}{4 x_{A} x_{p}}}{E_{A}^{2}+\frac{-q^{2}}{4 x_{A}^{2}}}\right)^{2}-\frac{1}{3} \frac{m_{N}^{2}+\frac{-q^{2}}{4 x_{p}^{2}}}{E_{A}^{2}+\frac{-q^{2}}{4 x_{A}^{2}}}\right) \\
& \left.\times \frac{x_{p}^{a}\left(1-x_{p}\right)^{b}+\mathbb{C}\left(\frac{m_{N}^{2}}{-q^{2}}\right)^{\frac{1}{2}} \frac{1}{x_{p}^{c}}}{x^{a}(1-x)^{b}+\mathbb{C}\left(\frac{m_{N}^{2}}{-q^{2}}\right)^{\frac{1}{2}} \frac{1}{x^{c}}}\right]
\end{aligned}
$$

where we made use of the nucleon on-mass shell. However, in the Thomas-Fermi approximation of Sec. II B, the nucleons are trapped in a potential well of depth $V_{0}=-40 \mathrm{MeV}$. Here, this will be enforced on average through the substitution $E_{p} \rightarrow E_{p}+V_{0}$. Finally, we note that the conformal scaling factor in Eq. (3.7) drops in the ratio in Eq. (3.9). So the key feature of strong coupling in Eq. (3.9) is the shift of the $\mathrm{x}$-distribution towards $x=1$ with no evolution needed.

In Fig. 2 we show the R-ratio (3.9) for $-q^{2} / m_{N}^{2}=25$ and $\tau=3$, with $\mathbb{C}=0$ for large $\mathrm{x}$. The surface parameters will be set to $\epsilon_{A}=0.1 / A^{\frac{1}{3}}$ and $\kappa_{A}=0$. Other choices of parameters are possible. The upper solid-red curve is for $A=42$, and the lower solid-blue curve is for $A=12$. The dashed curves are the high performance computing parametrization of the available nuclear parton distributions from [14]. The upper dashed-blue curve is for $A=12$, and the lower dashed-red curve is for $A=42$. We have limited

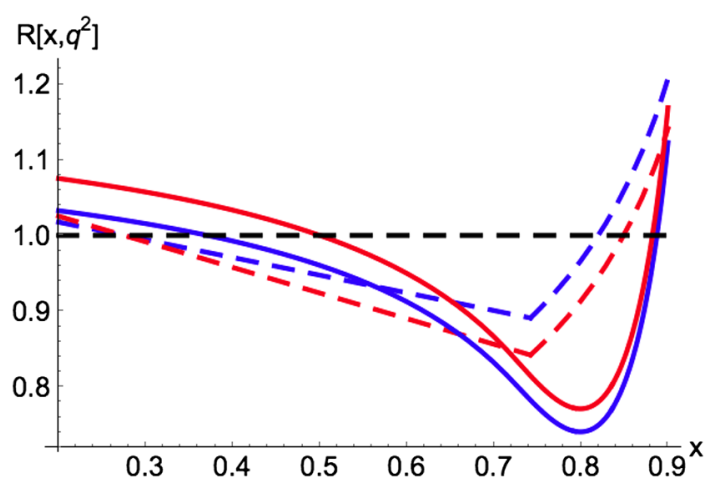

FIG. 2. R-ratio at large $x$ using the leading density contribution (3.9) and the holographic nucleon structure function (3.7) (solid curves), versus the parametrized empirical ratio from [14] (dashed curves), for $A=12$ (blue curves) and $A=42$ (red curves). 
the comparison to light nuclei since the calculation was restricted to the leading density contribution. Overall, Eq. (3.9) supports a depletion of valence partons at intermediate $\mathrm{x}$ and their rise due to Fermi motion at large $\mathrm{x}$. Without the binding energy, the depletion at intermediate $\mathrm{x}$ is constant. The depletion appears stronger for lighter nuclei in our analysis, with most of the A-dependence stemming from $\epsilon_{A}$. We expect this to change if a more realistic wave function for the finite nucleus is chosen with A-dependent binding energies [6].

The shadowing-antishadowing effects at low $\mathrm{x}$ are likely due to the combination of the low-x contribution in the nucleon structure function, together with coherent DIS scattering on a two-nucleon state smeared by Fermi motion. This latter effect requires the holographic derivation of the structure function on a two-nucleon-like state similar to the deuteron in holography, which is outside the scope of this work. We recall that the low-x regime in the extreme case of coherent scattering is captured in holography by scattering on an extremal Reissner-Nordström-AdS black hole in leading order, as we discussed recently in [15]. In a way, this corresponds to our expansion restricted to a single term with forward Compton scattering coherently on one A-nucleon charged state.

Finally, we would like to point out that in the perturbative regime, the nucleon (PDF) and nuclear (nPDF) parton distribution functions are scheme dependent, i.e., they depend on the factorization $\mu_{F}$ and renormalization $\mu_{R}$ scales. In the DIS scheme for instance, $\mu_{F}=\mu_{R}$ is assumed. However, the structure functions are scheme independent provided that the hard part in the factorization kernel is evaluated using the same scheme. The holographic analysis provides a nonperturbative estimate for both the PDF and nPDF at a single but low scale, say, the proton mass. In this sense, their evolution to higher scale, if needed, should follow the DIS scheme.

\section{CONCLUSIONS}

We have outlined a general framework for the analysis of DIS scattering on a nucleus. It consists in a density expansion of the forward Compton amplitude on a nucleus, as a sum of Compton amplitudes over stable nucleon states smeared over the nuclear volume using the Thomas-Fermi approximation. We have used the holographic nucleon structure function with a hard wall, to analyze the leading order contribution to the R-ratio for DIS on nuclei with different atomic number $A$.

The leading result for the nucleus R-ratio is independent of the hard conformal scaling factor, and supports a depletion at intermediate $\mathrm{x}$ and an enhancement at large $\mathrm{x}$ which are the hallmarks of the EMC effect. The depletion appears to be stronger for lighter nuclei if only the surface effects are taken into account with the same binding for all nuclei. We expect this to change when a realistic A-dependence of the binding energy is taken into account, e.g., using a shell model.

A key feature of the holographic forward Compton scattering on the nucleus in the DIS kinematics is that in the leading density approximation the hard virtual photon scatters coherently off each nucleon by exciting it to high energy and therefore small size, without breaking it. As a result, the structure functions are observed to be shifted towards $x=1$, besides their conformal scaling. In the double limit of large number of colors and gauge coupling, scattering off a hard parton in a proton is the exception and not the rule due to the large splitting rate, a point also at the origin of the modified Coulomb law [16]. A large fraction of the parton energy is lost before it is even struck. This is not the case at weak coupling, where scattering off a hard parton of a small size is more likely. Despite this, various QCD scaling laws are reproduced at strong coupling including the hard parton-counting rules [12].

Finally, we note that the leading nucleon and subleading two-nucleon structure functions can be improved using a soft wall to account for Reggeization, or a fine-tuned dilaton potential to account for asymptotic freedom [17]. More importantly, the one- and two-nucleon structure functions can be borrowed from experiment, or extracted from first principles using current lattice simulations for the quasidistributions [18].

\section{ACKNOWLEDGMENTS}

This work was supported by the U.S. Department of Energy under Contract No. DE-FG-88ER40388.
[1] M. R. Adams et al. (E665 Collaboration), Phys. Rev. Lett. 68, 3266 (1992); M. R. Adams et al. (Fermilab E665 Collaboration), Phys. Lett. B 287, 375 (1992); M. R. Adams et al. (E665 Collaboration), Z. Phys. C 67, 403 (1995).

[2] P. Amaudruz et al. (New Muon Collaboration), Nucl. Phys. B441, 3 (1995).

[3] J. Gomez et al., Phys. Rev. D 49, 4348 (1994).
[4] A. C. Benvenuti et al. (BCDMS Collaboration), Phys. Lett. B 189, 483 (1987).

[5] J. Polchinski and M. J. Strassler, J. High Energy Phys. 05 (2003) 012.

[6] L. L. Frankfurt and M. I. Strikman, Phys. Rep. 160, 235 (1988); R. P. Bickerstaff and A. W. Thomas, J. Phys. G 15, 1523 (1989); M. Arneodo, Phys. Rep. 240, 301 (1994). 
[7] J. V. Steele, H. Yamagishi, and I. Zahed, Phys. Lett. B 384, 255 (1996); Phys. Rev. D 56, 5605 (1997).

[8] W. Weise, Phys. Rep. 13, 53 (1974).

[9] P. V. Ramana Murthy, C. A. Ayre, H. R. Gustafson, L. W. Jones, and M. J. Longo, Nucl. Phys. B92, 269 (1975).

[10] R. C. Brower, M. J. Strassler, and C. I. Tan, J. High Energy Phys. 03 (2009) 050; Y. Hatta, E. Iancu, and A. H. Mueller, J. High Energy Phys. 01 (2008) 026; C. A. Ballon Bayona, H. Boschi-Filho, and N. R. F. Braga, J. High Energy Phys. 03 (2008) 064; J. H. Gao and B. W. Xiao, Phys. Rev. D 80, 015025 (2009).
[11] N. R. F. Braga and A. Vega, Eur. Phys. J. C 72, 2236 (2012).

[12] S. J. Brodsky and G. R. Farrar, Phys. Rev. Lett. 31, 1153 (1973).

[13] J. Kuti and V. F. Weisskopf, Phys. Rev. D 4, 3418 (1971).

[14] K. J. Eskola, H. Honkanen, V. J. Kolhinen, and C. A. Salgado, Phys. Lett. B 532, 222 (2002).

[15] K. Mamo and I. Zahed, arXiv:1807.07969.

[16] E. Shuryak and I. Zahed, Phys. Rev. D 69, 046005 (2004).

[17] U. Gursoy, E. Kiritsis, L. Mazzanti, G. Michalogiorgakis, and F. Nitti, Lect. Notes Phys. 828, 79 (2011).

[18] X. Ji, Phys. Rev. Lett. 110, 262002 (2013). 